OPEN ACCESS

Edited by:

Xuelei Ma

Sichuan University, China

Reviewed by:

Gunes Esendagli,

Hacettepe University, Turkey

Mouldy Sioud,

Oslo University Hospital, Norway

*Correspondence:

Sawsan Rashdan

sawsan.rashdan@

utsouthwestern.edu

Specialty section:

This article was submitted to

Cancer Immunity

and Immunotherapy,

a section of the journal

Frontiers in Oncology

Received: 04 June 2021 Accepted: 31 August 2021 Published: 30 September 2021

Citation:

Burke M and Rashdan S (2021)

Management of Immune-Related

Adverse Events in Patients With

Non-Small Cell Lung Cancer.

Front. Oncol. 11:720759.

doi: 10.3389/fonc.2021.720759

\section{Management of Immune-Related Adverse Events in Patients With Non-Small Cell Lung Cancer}

\author{
Michael Burke and Sawsan Rashdan *
}

Department of Internal Medicine, Division of Hematology and Oncology, University of Texas Southwestern Medical Center, Dallas, TX, United States

With proven efficacy of the use of immunotherapy in almost all stages of NSCLC, immunotherapy toxicity has become a very important topic that requires immediate recognition and management. The diagnosis of toxicities associated with immunotherapy in lung cancer can be very challenging and often requires multidisciplinary effort. This mini review gives an overview of the diagnosis and management of immune-related adverse events that arise from using immunotherapy in NSCLC, as well as the potential biomarkers for its early identification and future directions.

Keywords: lung cancer, immunotherapy, toxicity, adverse events, steroids, checkpoint inhibitor

\section{INTRODUCTION}

With an estimated 228,820 new cases of lung cancer in 2020 and 135,720 anticipated lung cancer deaths comprising $22 \%$ of all cancer deaths in the United States, the burden of non-small cell lung cancer (NSCLC) as the most common type of lung cancer and its treatment has become extraordinary (1). Over the past two decades, the care of NSCLC has been revolutionized by the introduction of cancer immunotherapy. Since the initial publications in the management of progressive metastatic disease in Checkmate-057 (2), Checkmate-017 (3), and Keynote-010 (4), immunotherapy has increasingly dominated the management of NSCLC moving to the first-line setting in metastatic disease (5), then with the use in locally advanced disease after concurrent chemoradiation therapy (6) and now the anticipated involvement in the neoadjuvant space (7) (Table 1).

Currently approved agents for the management of NSCLC include an ever-growing list of immunomodulatory drugs such as pembrolizumab, atezolizumab, nivolumab, durvalumab, and ipilumumab. Unfortunately, the inevitable afterbirth of this revolution has been the recognition of immune-related adverse events (irAEs) of treatment and the need for management of this novel class of complications. Thankfully, the majority of these irAEs are of minor grade and may be treated symptomatically with continuation of treatment; however, due to the nature of immunotherapy, nearly every organ system may be affected and to lethal ends. As will be discussed in the following review, the incidence and severity of these effects in the management of NSCLC may vary depending on drug class, patient characteristics, combination with radiation therapy, and combination with targeted therapy as well as other immunomodulatory drugs. 
TABLE 1 | FDA-approved immunotherapy in lung cancer without target mutation.

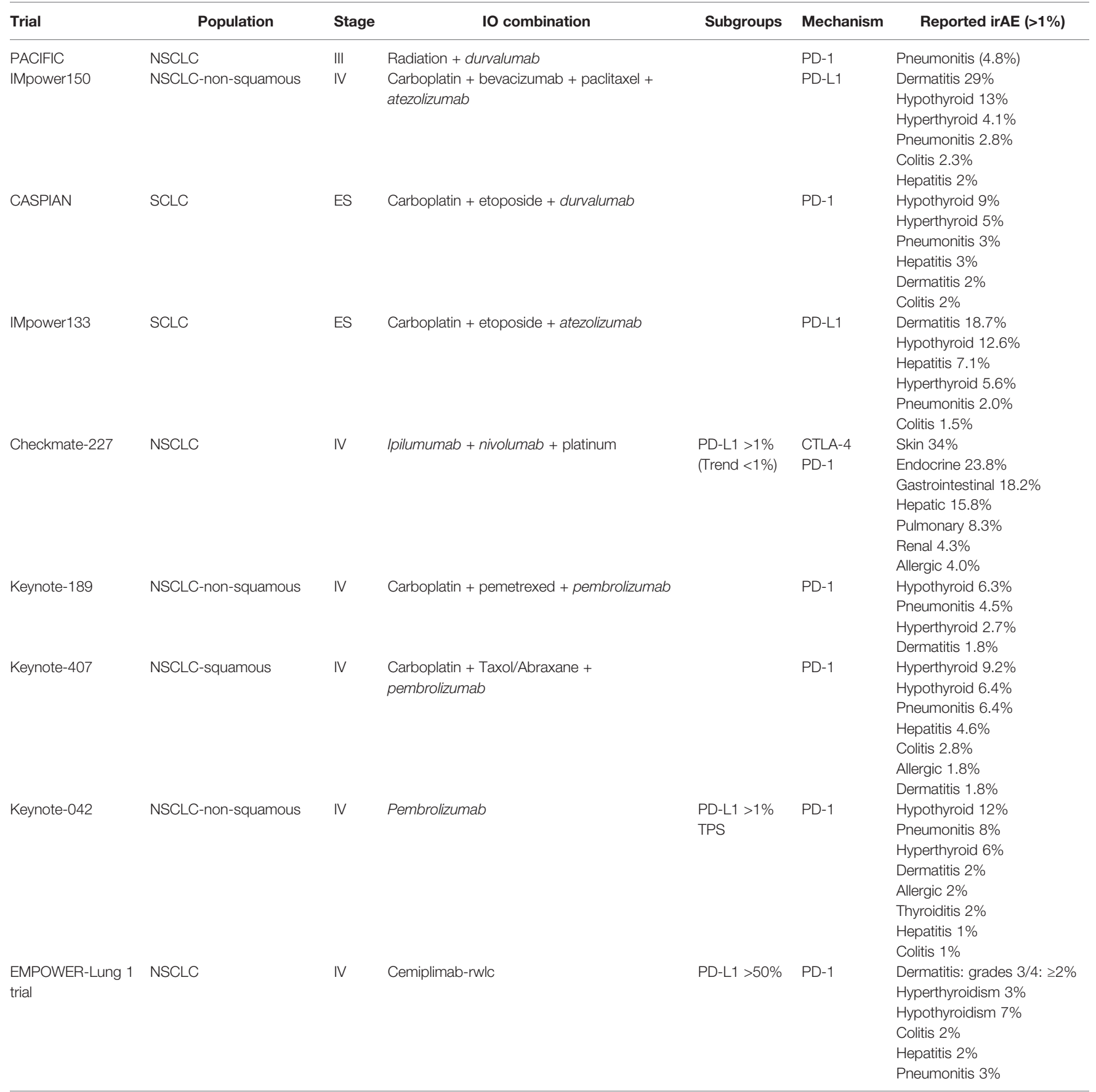

ES, Extensive stage; NSCLC, non-small cell lung cancer; SCLC, small cell lung cancer.

\section{DISCUSSION}

\section{Incidence}

\section{Pneumonitis}

The reported incidence of immune-related adverse events has varied since initial observations depending on the immune modulating agent and the clinical setting. Checkpoint inhibitor pneumonitis (CIP) currently occurs in $3 \%-5 \%$ of all cases; however, that estimate rises to $7 \%-13 \%$ in the setting of NSCLC treatment (8). As demonstrated in Checkmate-012, Checkmate-227, and Checkmate-568 (9-11), this incidence worsens when dual checkpoint inhibitor therapy with anticytotoxic T-lymphocyte-associated protein 4 (CTLA-4) and programmed death-1 (PD-1)/programmed death ligand-1 (PD-L1) is used (12). Furthermore, the increased incidence in NSCLC patients is attributable to the increased association of 
risk factors for immune-mediated pneumonitis in NSCLC patients including smoking, age $>70$ years, prior radiotherapy, prior lung disease (including chronic obstructive pulmonary disease), and exposure to the EGFR inhibitor osimertinib (8).

The interaction of checkpoint inhibitor therapy with osimertinib was first reported in the phase 1b TATTON trial assessing its tolerability in combination with durvalumab (13). The occurrence of clinically significant pneumonitis rose from $2.9 \%$ in the single agent arm to $38 \%$ in the combination arm resulting in the early termination of the trial. Interestingly, this observation remains to be replicated in the phase III multi-arm CAURAL trial (14). Furthermore, this interaction appears to be beyond concurrent treatment with an observed incidence of CIP with osimertinib administration during the first 3 months following checkpoint inhibitor treatment (15).

A similar interaction has been observed with concurrent or prior radiation treatment in NSCLC. In Keynote-001, 13\% of patients treated with pembrolizumab and history of prior radiotherapy exposure were observed to develop radiation recall pneumonitis over only $1 \%$ incidence in patients without radiation exposure. Comparable numbers were reported in the phase 2 DETERRED trial of concurrent radiotherapy with atezolizumab with an incidence of grade 2 or higher pneumonitis of $10 \%$. Additional observations suggest an association of checkpoint inhibitor pneumonitis with radiation dose with a nearly 9:1 ratio in patients treated with curativeintent radiotherapy over palliative-intent radiotherapy $(16,17)$.

\section{Colitis}

Inflammatory colitis following exposure to checkpoint inhibitor therapy represents another significant threat of morbidity and mortality in the management of NSCLC. A recent meta-analysis has demonstrated an overall incidence of $1.4 \%$ of colitis associated with immunomodulatory treatment, $0.89 \%$ for severe colitis, and $11.62 \%$ incidence of diarrhea in patients with NSCLC (18). Similar to the reports for CIP, the combination of CTLA-4 and anti-PD-1/ PD-L1 treatment increased the incidence from $0.89 \%$ of grade 3 colitis to $3 \%-5 \%$ for combination therapy (9). Moreover, an increased severity is associated with anti-CTLA-4 therapy in addition to an increased incidence of all extraintestinal manifestations including mouth ulcers, anal fissures, and esophagitis/gastritis (19).

Interestingly, while CIP has been observed with increased frequency in the first-line setting, inflammatory colitis appears to increase in incidence with subsequent lines of treatment (18). Furthermore, unlike CIP, no association has been observed between colitis and patient age, sex, smoking, and history of controlled autoimmune disease.

\section{Hepatitis}

Inflammatory hepatitis is an uncommon cause of treatment interruption in the management of NSCLC with estimates $<1 \%$ incidence of grade 3 hepatitis and 1\%-3\% of all grade hepatitis with the use of anti-PD-1/PD-L1 treatment in NSCLC $(20,21)$. The incidence has been shown to increase dramatically with combination therapy and/or the presence of liver metastases $(10,22)$.

\section{Dermatitis}

Dermatologic toxicities are among the most common immunerelated adverse events encountered in daily practice when treating lung cancer with an estimated incidence of $44 \%$ following CTLA-4 inhibition and 34\% with PD-1/PD-L1 targeting treatment (23). Early data have previously suggested that similarity between tumor antigen and somatic epitopes within the skin and fascia may provide a mechanistic explanation for the occurrence of dermatologic events (24). Manifestation of dermatologic adverse events can vary widely in presentation from pruritus and a mild maculopapular rash to bullous pemphigoid or psoriasis flare and even case reports of fulminant Stevens-Johnson syndrome (25). As such, recent National Comprehensive Cancer Network (NCCN) recommendations include a careful dermatologic exam on all patients with planned immunomodulatory treatment to detect and manage any mild or early grade disease before provocation to flare.

\section{Endocrinopathy}

In Keynote-001 (26), 21\% of patients receiving pembrolizumab for the management of NSCLC experienced thyroid dysfunction requiring eventual supplementation. Subsequent clinical experience with immunotherapy of NSCLC has confirmed an estimated incidence of endocrine irAEs of less than $23 \%$ with the overwhelming majority involving the thyroid and rarely exceeding grade $2(27,28)$. Hypophysitis secondary to anti$\mathrm{PD}-1 / \mathrm{PD}-\mathrm{L} 1$ therapy is considered extremely rare and more frequently observed secondary to anti-CTLA-4 therapy (29-31). Interestingly, a recent meta-analysis of 38 randomized clinical trials comprising 7,551 patients who underwent checkpoint inhibitor immunotherapy found a consistent reduction in the incidence of thyroiditis and insulin-deficient diabetes for single-agent anti-PD-1/PD-L1 when compared with anti-CTLA4 monotherapy $(28-30,32)$. As observed previously, the incidence of all immune-related endocrinopathies was higher with combination therapy $(28,33)$. Interestingly, the genetic risk for hypothyroidism was associated with risk of developing thyroid immune-related adverse events in NSCLC (34). Furthermore, the occurrence of gastrointestinal, dermatological, and endocrine irAEs in lung cancer patients has been proven to be a predictor of enhanced immune checkpoint inhibitor efficacy (35).

\section{Diagnosis, Treatment, and Follow-Up Checkpoint Inhibitor Pneumonitis}

Diagnosis of checkpoint inhibitor pneumonitis requires a high index of suspicion given the lack of specificity in the presenting symptoms including dyspnea, chest pain, cough, and fever (36). As such, a broad differential diagnosis exists in lung cancer patients including pneumonia, progression of disease, COPD exacerbation, pulmonary embolism, and radiation recall pneumonitis (37). Accordingly, appropriate workup may vary depending on clinical presentation; however, high-resolution computed topography (CT) scan is often useful and is recommended as one of the initial diagnostic tests performed in this setting. In most cases, a multidisciplinary approach is 
needed for accurate diagnosis. Pulmonary consultation for bronchoscopy with fungal and mycobacterial studies may be considered (38) (Table 2).

Regardless of the workup, CIP remains a diagnosis of exclusion, and a stepwise approach to empiric treatment guided by clinical presentation as defined by the CTCAE grading has gained favor (39). Grade 1 CIP presents asymptomatically involving less than $25 \%$ of available lung and discovered on surveillance imaging. Accordingly, for grade $1 \mathrm{CIP}$, a hold of immunotherapy for 3-4 weeks is recommended, and no steroid therapy is needed. Development of dyspnea without oxygen requirement is consistent with grade 2 CIP, and steroid therapy should be initiated with prednisone $1-2 \mathrm{mg} / \mathrm{kg} /$ day and tapered over 4-8 weeks. Severe dyspnea with associated hypoxia and involvement of $>50 \%$ of lung volume on imaging or persistence of grade 2 symptoms for $48 \mathrm{~h}$ despite steroid treatment requires escalation of immunosuppressive treatment most commonly with anti-TNF $\alpha$ therapy of infliximab at $5 \mathrm{mg} / \mathrm{kg}$ at 0,2 , and 6 weeks $(38,40)$.

Data for alternative treatment of grade 3 CIP are limited; however, there are encouraging early data for the use of tocilizumab with a $79 \%$ response rate $(36,41)$. Additional discussion regarding the use of mycophenolate mofetil and pooled intravenous immunoglobulin persists, but supportive data remain elusive.

\section{Colitis}

Management of gastrointestinal toxicity of checkpoint inhibitor therapy often follows a similar algorithmic approach based on clinical presentation (42). Traditional inflammatory bowel disease markers including C-reactive protein (CRP), calprotectin, and albumin have similarly failed to demonstrate an ability to predict the course of immune colitis from checkpoint inhibitor therapy. Endoscopic and histological assessment in the form of Mayo (43), UCEIS (44), and Nancy scores (42) have shown early promise in predicting the need for aggressive immunosuppression to avoid eventual colectomy.

The differential diagnosis of diarrhea and colitis following initiation of checkpoint inhibitor therapy is largely restricted to inflammatory disease, ischemic colitis, and infectious colitis. Endoscopy and directed biopsy may assist in guided initial therapy; however, consideration of infectious etiologies is crucial and a limited workup including stool ova and parasite assay, Clostridium difficile polymerase chain reaction (PCR), stool culture, and cytomegalovirus (CMV) serology should be considered in all patients with moderate to severe diarrhea and colitis.

Early grade 1 diarrhea of $<4$ stools per day may be treated symptomatically with anti-diarrheal medication and fluid replacement. If diarrhea increases to 4-6 stools per day or persists for more than 14 days, immunomodulatory treatment should be held, oral prednisone started at $0.5-1 \mathrm{mg} / \mathrm{kg} / \mathrm{day}$, and referral placed for outpatient colonoscopy. Clinical worsening with diarrhea of more than 7 stools per day and/or severe abdominal pain with evidence of peritonitis necessitates hospitalization for resuscitation, intravenous corticosteroids, and initiation of infliximab. Administration of anti-tumor necrosis factor- $\alpha$ therapy has been a mainstay of grade 3-4 treatment; however, a recent case series of seven patients demonstrated effective treatment by targeting gastrointestinal specific integrin with vedolizumab with an observed response in all patients (45).

\section{Hepatitis}

A broad differential diagnosis exists for the onset of clinically significant transaminitis following initiation of cancer immunotherapy, including infection, autoimmune hepatitis, and drug-induced liver injury. To that end, an expansive workup should be entertained for CMV, herpes simplex virus (HSV), parvovirus, adenovirus, Epstein-Barr virus (EBV), antiantinuclear antibody (ANA), anti-smooth muscle antibody (ASMA), anti-liver kidney microsomal type 1 antibody (LKM1), quantitative immunoglobulins, an abdominal ultrasound, and often liver biopsy $(46,47)$.

As the majority of cases are asymptomatic, early intervention is guided by laboratory findings of transaminitis. Of note, mild transaminitis with either AST or ALT below 3 times upper limit of normal (ULN) or total bilirubin below 1.5 times the ULN may be monitored with continuation of therapy. For grade 2 hepatitis with transaminases below 5 times ULN and total bilirubin below 3 times ULN, therapy is held and transaminases are monitored biweekly until levels return to grade 1 or below. Severe hepatitis with transaminases exceeding prior thresholds or evidence of liver failure requires immediate admission for intravenous corticosteroids of methylprednisolone $0.5-1.0 \mathrm{mg} / \mathrm{kg} /$ day and consideration of mycophenolate mofetil 500-1,000 mg Q12H if no improvement is observed within $72 \mathrm{~h}(46,48)$.

Historically, anti-TNF $\alpha$ therapy has been discouraged in severe transaminitis secondary to immunomodulatory treatment with the standard escalation to mycophenolate for steroid refractory disease. Here, again, alternative treatments may be considered in the appropriate clinical context with common options including tacrolimus $0.1-0.15 \mathrm{mg} / \mathrm{kg} /$ day or anti-thymocyte globulin $1.5 \mathrm{mg} / \mathrm{kg} /$ day with consideration of hepatology consultation (49).

\section{Dermatitis}

Due to the wide variety in dermatologic presentation, an algorithmic approach should be taken in the majority of cases encountered in clinical practice with involvement of specialty care for additional workup and management (50). A mild rash involving $<10 \%$ body surface area (BSA) with mild symptoms of burning or pruritus may be managed appropriately with mediumto high-potency topical corticosteroids and symptomatic care of oral anti-histamine treatment. Progression to grade 2 rash involving 10\%-30\% BSA with symptoms inhibiting instrumental activities of daily living would be a reasonable indication for the addition of systemic corticosteroids with prednisone $0.5-1 \mathrm{mg} / \mathrm{kg} /$ day with consideration of checkpoint inhibitor hold. Inpatient care and urgent dermatologic consultation may be considered for rashes involving more than 30\% BSA depending on severity of symptoms. Provider discretion in addition to patient discussion is 
TABLE 2 | Common irAE treatment algorithm.

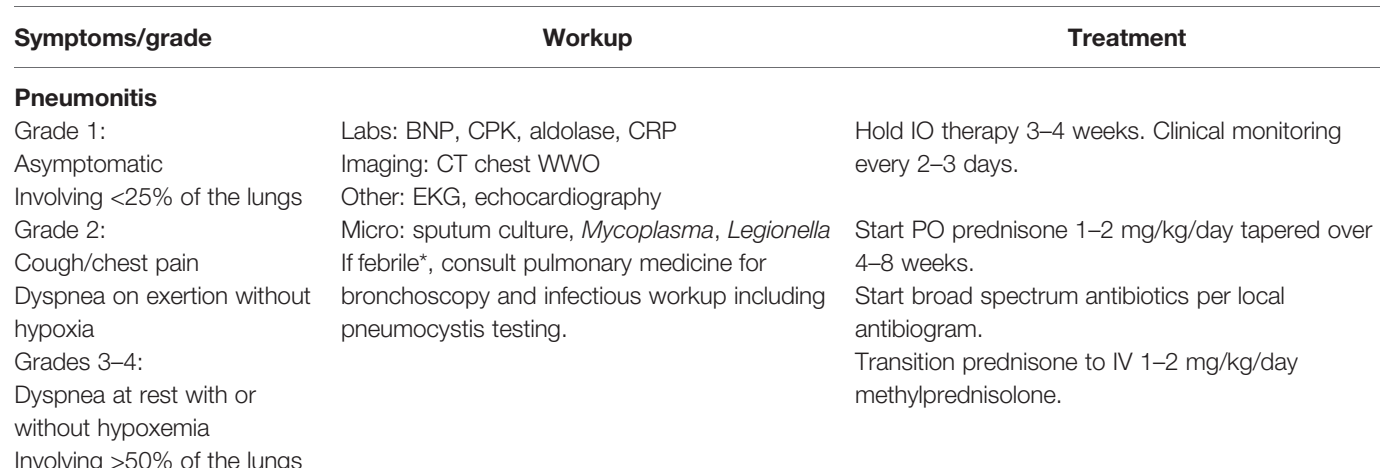

Follow-up/monitoring

If persistent, escalate treatment.

If unimproved after $48 \mathrm{~h}$, escalate treatment.

If unimproved at $48 \mathrm{~h}$, start infliximab at $5 \mathrm{mg} / \mathrm{kg}$ on days 0 , 15 , and 43.

${ }^{\star \star}$ Permanent discontinuation of immunotherapy.

\section{Alternative agents:}

\section{Mycrophenolate mofetil BID}

IVIG

\section{Tocilizumab}

\section{Colitis}

\section{Grade 1:}

$<4$ liquid stools above daily baseline

Grade 2:

4-6 liquid stools above daily baseline or new abdominal pain/hematochezia

Grades 3-4:

$>7$ liquid stools above daily baseline, life-threatening

\author{
Labs: CBC, CMP, TFTs, CRP \\ Other: fecal fat \\ Micro: stool culture, ova/parasites, CMV PCR, \\ Cdiff PCR, cryptosporidia \\ *If persistent, consider Gl referral for \\ colonoscopy or hematochezia. \\ **If peritoneal signs, low threshold CT \\ abdomen WWO and urgent surgical \\ consultation.
}

Continue $1 \mathrm{O}$ with symptomatic treatment of loperamide and fluid repletion.

Start PO prednisone $0.5-1 \mathrm{mg} / \mathrm{kg} /$ day *do not wait for colonoscopy.

Transition to IV methylprednisolone 1-2 mg/kg/daily. Urgent Gl consultation.
If persistent for $>14$ days or worsening escalate treatment.

Clinical monitoring every $72 \mathrm{~h}$, if worsening escalate treatment.
If unimproved at $72 \mathrm{~h}$, start infliximab at $5 \mathrm{mg} / \mathrm{kg}$ on days 0 , 15 , and 43.

\section{Alternative agents: \\ Mycophenolate mofetil BID \\ Tacrolimus}

${ }^{* *}$ Hold escalation of immunosuppression until colonoscopy/sigmoidoscopy is performed.

\section{Hepatitis}

Grade 1:

AST and ALT $<3 x$ ULN

Total bilirubin $<1.5 x$ ULN

Grade 2:

AST and/or ALT $<5 x$ ULN

Total bilirubin $<3 x$ ULN

Grades 3-4:

\begin{abstract}
Labs: anti-ANA/SMA/LKM/SLA/LP, iron panel, $\quad$ Continue $I O$.
\end{abstract}
quantitative Igs

Micro: hepatitis A/B/C, HIV, parvovirus, CMV,

HSV

Other:

Imaging: liver US W Doppler

*Consider hepatology consult and imaging-

guided biopsy if worsening with initial management.
Start prednisone 1 mg/kg/day.

Transition to IV methylprednisolone 2 mg/kg/day.

${ }^{\star *}$ Permanent discontinuation of immunotherapy.

Repeat CMP weekly.

Monitor LFTs with INR and albumin biweekly. Escalate management if worsening. If no response or worsening at $48 \mathrm{~h}$, start mycophenolate mofetil 500-1,000 mg BID.

\section{Alternative agents:}

**Second-line agents for refractory disease include tacrolimus and anti-thymocyte globulin.

\section{Dermatitis}

Grade 1:

Mild rash involving $<10 \%$

BSA

Grade 2:

Symptomatic involving 10\%30\% BSA

Physical examination and history excluding other common causes including viral exanthema and drug rash.

${ }^{*}$ Consider urgent referral to dermatology and punch biopsy if refractory to moderate topical steroids or severe symptoms.

Grade 3:

Involving $>30 \%$ BSA or severe symptoms.

Avoid irritants. Consider mild strength topical corticosteroids and PRN oral antihistamine.

Escalation to moderate-/high-intensity topical corticosteroids and/or initiation of $\mathrm{PO}$ prednisone $0.5-1 \mathrm{mg} / \mathrm{kg}$ daily.

Hold IO for severe symptoms and consider admission or IV methylprednisolone at $1-2$ mg/kg/ day and urgent dermatology evaluation.

Alternative agents:

***Pregabalin, gabapentin, and aprepitant can be considered for management of refractory pruritus.

Additional considerations:

*With prolonged steroid management, calcium/vitamin D supplementation, pneumocystis prophylaxis, and acid suppression.

${ }^{* *}$ Avoid infliximab if evidence of hepatic injury. 
critical as many grade 3 rashes with mild symptoms may be reasonably managed in the outpatient setting.

Special consideration should be given to alternative management of checkpoint inhibitor-induced pruritus with gabapentin, pregabalin, and/or aprepitant in cases refractory to antihistamine treatment (51). Consultation of dermatology and disease-directed care should be strongly considered for all cases of grade 4 adverse events including but not limited to drug rash with eosinophilia and systemic symptoms (DRESS) syndrome, toxic epidermal necrolysis, and Steven-Johnson syndrome ahead of permanent discontinuation of checkpoint inhibitor therapy.

\section{Endocrinopathies}

With the availability of screening assays for many of the observed immune-related endocrine complications of treatment, many are caught early in disease course. Accordingly, in addition to vital signs, routine screening with a basic metabolic panel, calcium, parathyroid hormone (PTH), thyroid stimulating hormone (TSH), free T4, adrenocorticotropic hormone (ACTH), and/or AM cortisol should be obtained ahead of every cycle for the first 6 months and progressively spaced thereafter $(33,50)$. Otherwise, a high degree of clinical suspicion should be employed for patients undergoing immunotherapy with new or worsening symptoms including fatigue, headache, confusion, diplopia, nausea, vomiting, weakness, weight gain, constipation, diarrhea, sweating, weight loss, polyuria, polydipsia, paresthesia, muscle cramps, lightheadedness, tachycardia, bradycardia, and hypotension (50). Additional workup and management should be guided appropriately with endocrinology consultation for any patient found to be symptomatic or with a positive screen.

In contrast to many other immune-related adverse events, management of endocrinopathy is focused on hormone repletion rather than escalation of immunosuppression and reversal of disease course. Though rare, recognition and diagnosis of adrenal insufficiency is of critical importance for the prevention of adrenal crisis. In the absence of screening, these patients may present with headache, confusion, fatigue, nausea, vomiting, weight loss, and double vision with additional workup directed to explore primary and secondary adrenal insufficiency. With an elevated ACTH with or without hyponatremia and hyperkalemia indicative of primary adrenal insufficiency, additional workup should include an abdominal CT, plasma renin, and 21-hydroxylase antibody serology while administering empiric treatment. In the setting of a depressed ACTH, a pituitary magnetic resonance imaging (MRI), visual field exam, and laboratory workup including prolactin, follicle-stimulating hormone (FSH), luteinizing hormone ( $\mathrm{LH})$, estradiol, and testosterone should be entertained.

Asymptomatic or minimally symptomatic patients can be started on oral hydrocortisone $15-25 \mathrm{mg}$ daily in two to three divided doses or oral fludrocortisone $100 \mathrm{mcg}$ daily. Moderately to severely symptomatic patients should be hospitalized for intravenous glucocorticoids of hydrocortisone with $100 \mathrm{mg}$ bolus upfront and then $50 \mathrm{mg}$ every $6 \mathrm{~h}$ in addition to aggressive fluid resuscitation with normal saline and thyroid hormone repletion.

\section{Rare}

Inflammatory arthritis is an increasingly recognized complication of lung cancer immunotherapy with incidence ranging from $1 \%$ to $7 \%(52-54)$. When suspected, initial workup begins with physical exam and documentation of all involved inflamed joints in addition to laboratory workup of ANA, rheumatoid factor (RF), cyclic citrullinated peptide (CCP) antibodies, and human leukocyte antigen (HLA)-B27 as well as plain films of the involved joints. For mild cases without interruption of activities of daily living, symptomatic care can be pursued with oral prednisone $10-20 \mathrm{mg}$ daily and non-steroidal anti-inflammatory drugs (NSAIDs) as needed for pain relief for 4-6 weeks of therapy with concurrent serial examinations (52). For more severe or refractory cases, additional workup and management should be pursued in coordination with rheumatology referral with consideration of immunotherapy hold.

The incidence of renal toxicity has been reported at $<1 \%$ for single-agent therapy; however, it has been reported to be as high as $5 \%$ with the combination of CTLA-4 and PD-1/PD-L1 therapy (55). Mild elevations in serum creatinine less than $1.5 \times$ baseline can be observed with appropriate outpatient hydration; however, elevation above $1.5 \times$ baseline should prompt the hold of immunotherapy in addition to consideration for urgent resuscitation and workup with nephrology consultation depending on the degree of renal insufficiency (55). Immune-related neurologic, ophthalmic, and cardiac toxicities are exceedingly rare with reported incidences often $<1 \%$ with management best guided by subspecialty consultation $(50,56)$.

\section{Prediction Biomarker Research}

Unfortunately, to date, there are no clinically useful predictive biomarkers to assess immune-related adverse event development in daily practice (57). The association of germline genetic variation with the risk for developing immune-related adverse events when using checkpoint inhibitors is still unclear. Variants in the major histocompatibility complex (MHC) locus were found to be strongly associated with autoimmune diseases in humans (58). Given the strong influence of these genetic variants on autoimmunity, looking at genome-wide single nucleotide polymorphism (SNP) data that are collected from patients treated with checkpoint inhibitors can help in recognizing variants that are associated with irAE. Furthermore, this can help in developing individual polygenic risk scores that can provide a personalized score that measures the genetic risk for an irAE (59). Several retrospective case series have identified discrete class II HLA alleles correlated with the development of several immune-related adverse events ranging from inflammatory arthritis to diabetes mellitus and adrenal insufficiency to colitis (60-65). While these alleles have been associated with the development of immune-related adverse events, they are not wholly predictive; accordingly, their clinical utility even when available remains uncertain.

Interestingly, early diversification of the circulating CD4+ and CD8+ T-cell repertoires following initiation of anti-CTLA-4 treatment has been associated with the onset of immune-related adverse events (66-69). Again, while hypothesis generating, the 
practical utility of these data remains elusive and is likely outweighed by the burden of recurrent assessment to an uncertain end. Alternatively, serological markers such as surfactant protein, transforming growth factor $\beta 1$, tumor necrosis factor- $\alpha$, interleukin $1 \beta$, and interleukin 6 for the prediction of radiation-induced pneumonitis have been studied extensively, and their significance toward immunotherapyinduced pulmonary toxicity remains uncertain (70-72).

\section{Rechallenge of CPI}

One of the most pressing questions facing the management of cancer patients undergoing immunomodulatory treatment is the possibility of rechallenge following the occurrence of an immune-related adverse event. With the majority of immunerelated adverse events manifesting as low severity grade 1-2 disease, a recent consensus statement from the Society for Immunotherapy of Cancer asserts that rechallenge is reasonable following resolution of event and completion of planned therapy (73). More controversial is the discussion of rechallenge in patients who have undergone a grade 3 event; here, guidance has been largely left to a personalized risk/benefit discussion between patient and provider.

Previously, in a retrospective study, 482 patients undergoing anti-PD-L1 immunomodulatory therapy and suffering treatment interruption secondary to a grade $2 / 3$ immune-related adverse event were observed for possible recurrence on rechallenge (74). Interestingly, while $26 \%$ experienced recurrence of the same adverse event and $23 \%$ suffered an entirely new immune-related adverse event, $51 \%$ of patients did not suffer a recurrent event. A similar occurrence of subsequent events was observed regardless of grade on initial onset, but it did correlate time of initial onset with those events occurring with 3 months of treatment initial most likely to recur.

Further complicating the discussion of rechallenge is the correlation of immune-related adverse event occurrence with disease response. Despite early data suggesting that the occurrence of irAE was predictive of disease response, subsequent studies failed to confirm the initial observation (75-77). Recent data further suggest that early treatment of immune-related adverse events may improve overall survival of those undergoing immunomodulatory treatment by allowing rechallenge and prolonged disease control $(74,76,78)$.

\section{Pre-Existing Autoimmune Disease}

Management of lung cancer patients with an indication for checkpoint inhibitor therapy and a history of pre-existing autoimmune disease is an additional point of ongoing debate (79). As a measure to minimize confounding bias, patients with a known history of active autoimmune disease have been excluded from large randomized control trials of immunomodulatory therapy limiting the availability of high-quality data in this population $(57,80)$. Available retrospective case series assessing anti-CTLA-4 treatment with pre-existing autoimmune disease has emerged in the melanoma literature with a trend toward increased occurrence and severity of irAE when compared with historical controls $(81,82)$.
Conversely, limited case series suggest that the risk of irAE occurrence with anti-PD-1/PD-L1 therapy in the setting of preexisting autoimmune disease is comparable to those patients without known history and without identifiable compromise in efficacy $(77,83-85)$. While these data are encouraging, not all autoimmune conditions bear the same risk of morbidity and mortality on flare. As such, special consideration must be applied to patients with histories of life-threatening autoimmune diseases involving the neurologic and neuromuscular systems such as myasthenia gravis (57). Moreover, in a recent large retrospective cohort, immunosuppression with $10 \mathrm{mg}$ or more of daily prednisone was associated with statistically significant decreases in response rate, progression-free survival, and overall survival for NSCLC patients on anti-PD-1 therapy (86).

Current summary recommendations from the NCCN suggest careful consideration of checkpoint inhibitor therapy in appropriate patients with well-controlled autoimmune disease requiring low to no immunosuppression in coordination with appropriate subspecialty care.

\section{CONCLUSION}

It is now clear that we need to understand and deal with the respiratory effects of a range of cancer treatments. Although much has been learned regarding the management of immunerelated adverse events since their introduction into the NSCLC population, several outstanding questions remain. The lack of reliable, clinically deployable predictive biomarkers and patient characteristics to predict autoimmune development remains an area of active need. Such an assay would allow for the tailored treatment of every patient maximizing the probability of response while minimizing the occurrence of autoimmune phenomena and, thus, harm of treatment. Additional comparative work regarding the incidence of autoimmune events between immunomodulatory classes might partially address this need with lower barrier to entry. Currently, several active clinical trials are addressing this need investigating the correlation of autoantibody and other serological changes in immunotherapy patients with significant adverse event occurrence (NCT03984318, NCT03868046, NCT03409016).

Moreover, as discussed regarding the management of checkpoint inhibitor colitis, while many of the developed treatment algorithms stratify based on universal CTCAE criteria, this often has little correlation with eventual severity of disease, escalation of treatment, duration of treatment, and interruption of immunomodulatory therapy. Additional investigative collaboration across specialties will be required to address this need possibly by the translation of extant tools for the management of known autoimmune disease. Lastly, while anti-TNF $\alpha$ therapy in the form of infliximab has emerged as a rational and consensus standard of care for many forms of steroid refractory disease, often high-quality data remain lacking. To that end, trials investigating novel agents as well as traditional immunosuppressive therapy are ongoing (NCT04375228, NCT04552704). 
Thankfully, the overwhelming majority of immune-related adverse events secondary to checkpoint inhibitor therapy appear to be of minor grade with only brief interruptions in treatment if any. Furthermore, with prompt recognition, an algorithmic approach as outlined here and by prior groups can achieve appropriate disease control.

\section{REFERENCES}

1. Data S. (2020).

2. Horn L, Spigel DR, Vokes EE, Holgado EH, Ready N, Steins M, et al. Nivolumab Versus Docetaxel in Previously Treated Patients With Advanced Non-Small-Cell Lung Cancer: Two-Year Outcomes From Two Randomized, Open-Label, Phase III Trials (CheckMate 017 and CheckMate 057). J Clin Oncol (2017) 35(35):3924-33. doi: 10.1200/JCO.2017.74.3062

3. Reck M, Taylor F, Penrod JR, DeRosa M, Morrissey L, Dastani H, et al. Impact of Nivolumab Versus Docetaxel on Health-Related Quality of Life and Symptoms in Patients With Advanced Squamous Non-Small Cell Lung Cancer: Results From the CheckMate 017 Study. J Thorac Oncol (2018) 13 (2):194-204. doi: 10.1016/j.jtho.2017.10.029

4. Herbst RS, Garon EB, Kim DW, Cho BC, Perez-Gracia JL, Han J-Y, et al. Long-Term Outcomes and Retreatment Among Patients With Previously Treated, Programmed Death-Ligand 1positive, Advanced NonSmall-Cell Lung Cancer in the KEYNOTE-010 Study. J Clin Oncol (2020) 38 (14):1580-90. doi: 10.1200/JCO.19.02446

5. Reck M, Rodríguez-Abreu D, Robinson AG, Hui R, Csőszi T, Fülöp A, et al. Pembrolizumab Versus Chemotherapy for PD-L1-Positive Non-Small-Cell Lung Cancer. N Engl J Med (2016) 375(19):1823-33. doi: 10.1056/ NEJMoa1606774

6. Uemura T, Hida T. Durvalumab Showed Long and Durable Effects After Chemoradiotherapy in Stage III Non-Small Cell Lung Cancer: Results of the PACIFIC Study. J Thorac Dis (2018) 10(Suppl 9):S1108-12. doi: 10.21037/ jtd.2018.03.180

7. Shu CA, Gainor JF, Awad MM, Chiuzan C, Grigg CM, Pabani A, et al. Neoadjuvant Atezolizumab and Chemotherapy in Patients With Resectable Non-Small-Cell Lung Cancer: An Open-Label, Multicentre, Single-Arm, Phase 2 Trial. Lancet Oncol (2020) 21(6):786-95. doi: 10.1016/S1470-2045 (20)30140-6

8. Zhai X, Zhang J, Tian Y, Li J, Jing W, Guo H, et al. The Mechanism and Risk Factors for Immune Checkpoint Inhibitor Pneumonitis in non-Small Cell Lung Cancer Patients. Cancer Biol Med (2020) 17(3):599-611. doi: 10.20892/ j.issn.2095-3941.2020.0102

9. Hellmann MD, Rizvi NA, Goldman JW, Gettinger SN, Borghaei H, Brahmer JR, et al. Nivolumab Plus Ipilimumab as First-Line Treatment for Advanced Non-Small-Cell Lung Cancer (CheckMate 012): Results of an Open-Label, Phase 1, Multicohort Study. Lancet Oncol (2017) 18(1):31-41. doi: 10.1016/ S1470-2045(16)30624-6

10. Reck M, Ciuleanu TE, Lee J-S, Schenker M, Audigier-Valette C, Zurawski B, et al. First-Line Nivolumab Plus Ipilimumab Versus Chemotherapy in Advanced NSCLC With 1\% or Greater Tumor PD-L1 Expression: PatientReported Outcomes From CheckMate 227 Part 1. J Thorac Oncol (2021) 16 (4):665-76. doi: 10.1016/j.jtho.2020.12.019

11. Ready N, et al. First-Line Nivolumab Plus Ipilimumab in Advanced NonSmall-Cell Lung Cancer (CheckMate 568): Outcomes by Programmed Death Ligand 1 and Tumor Mutational Burden as Biomarkers. J Clin Oncol (2019) 37 (12):992-1000. doi: 10.1200/JCO.18.01042

12. Cadranel J, Canellas A, Matton L, Darrason M, Parrot A, Naccache J-M, et al. Pulmonary Complications of Immune Checkpoint Inhibitors in Patients With Nonsmall Cell Lung Cancer. Eur Respir Rev (2019) 28(153):190058. doi: 10.1183/16000617.0058-2019

13. Oxnard GR, et al. TATTON: A Multi-Arm, Phase Ib Trial of Osimertinib Combined With Selumetinib, Savolitinib, or Durvalumab in EGFR-Mutant Lung Cancer. Ann Oncol (2020) 31(4):507-16. doi: 10.1016/j.annonc.2020.01.013

14. Yang JC, et al. Osimertinib Plus Durvalumab Versus Osimertinib Monotherapy in EGFR T790M-Positive NSCLC Following Previous EGFR

\section{AUTHOR CONTRIBUTIONS}

All authors contributed to the article and approved the submitted version. UT Southwestern is aware of the submission and agrees to it. The corresponding author is responsible for submitting a competing financial interest statement on behalf of all authors of the paper.

TKI Therapy: CAURAL Brief Report. J Thorac Oncol (2019) 14(5):933-9. doi: 10.1016/j.jtho.2019.02.001

15. Schoenfeld AJ, Arbour KC, Rizvi H, Iqbal AN, Gadgeel SM, Girshman J, et al. Severe Immune-Related Adverse Events are Common With Sequential PD(L)1 Blockade and Osimertinib. Ann Oncol (2019) 30(5):839-44. doi: 10.1093/ annonc/mdz077

16. Voong KR, Hazell SZ, Fu W, Hu C, TingLin C, Ding K, et al. Relationship Between Prior Radiotherapy and Checkpoint-Inhibitor Pneumonitis in Patients With Advanced Non-Small-Cell Lung Cancer. Clin Lung Cancer (2019) 20(4):e470-9. doi: 10.1016/j.cllc.2019.02.018

17. Chen Y, Huang Z, Xing L, Meng X, Yu J. Radiation Recall Pneumonitis Induced by Anti-PD-1 Blockade: A Case Report and Review of the Literature. Front Oncol (2020) 10:561. doi: 10.3389/fonc.2020.00561

18. Lin LL, Lin G-F, Luo Q, Chen X-Q. The Incidence and Relative Risk of PD-1/ PD-L1 Inhibitors-Related Colitis in non-Small Cell Lung Cancer: A MetaAnalysis of Randomized Controlled Trials. Int Immunopharmacol (2019) 77:105975. doi: 10.1016/j.intimp.2019.105975

19. Rocha M, Correia de Sousa J, Salgado M, Rocha A, Araújo I. Management of Gastrointestinal Toxicity From Immune Checkpoint Inhibitor. GE Port J Gastroenterol (2019) 26(4):268-74. doi: 10.1159/000494569

20. Herbst RS, Baas P, Kim D-W, Felip E, Pérez-Gracia JL, Han J-Y, et al. Pembrolizumab Versus Docetaxel for Previously Treated, PD-L1-Positive, Advanced non-Small-Cell Lung Cancer (KEYNOTE-010): A Randomised Controlled Trial. Lancet (2016) 387(10027):1540-50. doi: 10.1016/S0140-6736 (15)01281-7

21. Rizvi NA, Mazières J, Planchard D, Stinchcombe TE, Antonia MD, Horn MD, et al. Activity and Safety of Nivolumab, an Anti-PD-1 Immune Checkpoint Inhibitor, for Patients With Advanced, Refractory Squamous non-Small-Cell Lung Cancer (CheckMate 063): A Phase 2, Single-Arm Trial. Lancet Oncol (2015) 16(3):257-65. doi: 10.1016/S1470-2045(15)70054-9

22. Belli C, Bellia M, Zuin L, Mazzarella D, Trapani P, D'Amico E, et al. Liver Toxicity in the Era of Immune Checkpoint Inhibitors: A Practical Approach. Crit Rev Oncol Hematol (2018) 132:125-9. doi: 10.1016/j.critrevonc.2018.09.019

23. Si X, He C, Zhang L, Liu X, Li Y, Wang H, et al. Management of Immune Checkpoint Inhibitor-Related Dermatologic Adverse Events. Thorac Cancer (2020) 11(2):488-92. doi: 10.1111/1759-7714.13275

24. Tanaka R, Okiyama N, Okune M, Ishitsuka Y, Watanabe O, Furuta M, et al. Serum Level of Interleukin-6 is Increased in Nivolumab-Associated Psoriasiform Dermatitis and Tumor Necrosis Factor-Alpha Is a Biomarker of Nivolumab Recativity. J Dermatol Sci (2017) 86(1):71-3. doi: 10.1016/ j.jdermsci.2016.12.019

25. Hwang SJE, Park JJW, Wakade W, Chou S, Byth K, Fernandez-Penas P, et al. Cutaneous Adverse Events of Anti-Programmed Death 1 Antibodies Combined With Anti-Cytotoxic T-Lymphocyte-Associated Protein 4 Therapy Use in Patients With Metastatic Melanoma. Melanoma Res (2019) 29(2):172-7. doi: 10.1097/CMR.0000000000000518

26. Leighl NB, Hellmann MD, Hui R, Carcereny E, Felip E, Ahn M-J, et al. Pembrolizumab in Patients With Advanced Non-Small-Cell Lung Cancer (KEYNOTE-001): 3-Year Results From an Open-Label, Phase 1 Study. Lancet Respir Med (2019) 7(4):347-57. doi: 10.1016/S2213-2600(18)30500-9

27. Dudzinska M, Szczyrek K, Wojas-Krawczyk J, Świrska I, Chmielewska A, Zwolak A. Endocrine Adverse Events of Nivolumab in Non-Small Cell Lung Cancer Patients-Literature Review. Cancers (Basel) (2020) 12(8):2314. doi: $10.3390 /$ cancers 12082314

28. Barroso-Sousa R, Barry WT, Garrido-Castro AC, Hodi FS, Min L, Krop IE, et al. Incidence of Endocrine Dysfunction Following the Use of Different Immune Checkpoint Inhibitor Regimens: A Systematic Review and MetaAnalysis. JAMA Oncol (2018) 4(2):173-82. doi: 10.1001/jamaoncol.2017.3064 
29. Hodi FS, O'Day SJ, McDermott DF, Weber RW, Sosman JA, Haanen JB, et al. Improved Survival With Ipilimumab in Patients With Metastatic Melanoma. N Engl J Med (2010) 363(8):711-23. doi: 10.1056/NEJMoa1003466

30. Larkin J, Chiarion-Sileni V, Gonzalez R, Grob JJ, Cowey CL, Lao CD, et al. Combined Nivolumab and Ipilimumab or Monotherapy in Untreated Melanoma. N Engl J Med (2015) 373(1):23-34. doi: 10.1056/NEJMoa1504030

31. Torino F, Corsello SM, Salvatori R. Endocrinological Side-Effects of Immune Checkpoint Inhibitors. Curr Opin Oncol (2016) 28(4):278-87. doi: 10.1097/ CCO.0000000000000293

32. Mellati M, Eaton KD, Brooks-Worrell BM, Hagopian WA, Martins R, Palmer JP, et al. Anti-PD-1 and Anti-PDL-1 Monoclonal Antibodies Causing Type 1 Diabetes. Diabetes Care (2015) 38(9):e137-8. doi: 10.2337/dc15-0889

33. Stelmachowska-Banas M, Czajka-Oraniec I. Management of Endocrine Immune-Related Adverse Events of Immune Checkpoint Inhibitors: An Updated Review. Endocr Connect (2020) 9(10):R207-28. doi: 10.1530/EC20-0342

34. Luo J, Martucci VL, Quandt Z, Groha S, Murray MH, Lovly CM, et al. Immunotherapy-Mediated Thyroid Dysfunction: Genetic Risk and Impact on Outcomes With PD-1 Blockade in Non-Small Cell Lung Cancer. Clin Cancer Res (2021). doi: 10.1158/1078-0432.CCR-21-0921

35. Wang D, Chen C, Gu Y, Lu W, Zhan P, Liu H, et al. Immune-Related Adverse Events Predict the Efficacy of Immune Checkpoint Inhibitors in Lung Cancer Patients: A Meta-Analysis. Front Oncol (2021) 11:631949. doi: 10.3389/ fonc.2021.631949

36. Naidoo J, Wang X, Woo KM, Iyriboz D, Halpenny J, Cunningham JE, et al. Pneumonitis in Patients Treated With Anti-Programmed Death-1/ Programmed Death Ligand 1 Therapy. J Clin Oncol (2017) 35(7):709-17. doi: 10.1200/JCO.2016.68.2005

37. Wang H, Guo X, Zhou J, Li Y, Duan L, Si X, et al. Clinical Diagnosis and Treatment of Immune Checkpoint Inhibitor-Associated Pneumonitis. Thorac Cancer (2020) 11(1):191-7. doi: 10.1111/1759-7714.13240

38. Rashdan S, Minna JD, Gerber DE. Diagnosis and Management of Pulmonary Toxicity Associated With Cancer Immunotherapy. Lancet Respir Med (2018) 6(6):472-8. doi: 10.1016/S2213-2600(18)30172-3

39. Naidoo J, Nishino M, Patel SP, Shankar B, Rekhtman N, Illei P, et al. ImmuneRelated Pneumonitis After Chemoradiotherapy and Subsequent Immune Checkpoint Blockade in Unresectable Stage III Non-Small-Cell Lung Cancer. Clin Lung Cancer (2020) 21(5):e435-44. doi: 10.1016/ j.cllc.2020.02.025

40. Suresh K, RanhVoong K, Shankar B, Forde PM, Ettinger DS, Marrone KA, et al. Pneumonitis in Non-Small Cell Lung Cancer Patients Receiving Immune Checkpoint Immunotherapy: Incidence and Risk Factors. J Thorac Oncol (2018) 13(12):1930-9. doi: 10.1016/j.jtho.2018.08.2035

41. Stroud CR, Hegde A, Cherry C, Naqash AR, Sharma N, Addepalli S, et al. Tocilizumab for the Management of Immune Mediated Adverse Events Secondary to PD-1 Blockade. J Oncol Pharm Pract (2019) 25(3):551-7. doi: $10.1177 / 1078155217745144$

42. Cheung VTF, Gupta T, Olsson-Brown A, Subramanian S, Sasson SC, Heseltine S, et al. Immune Checkpoint Inhibitor-Related Colitis Assessment and Prognosis: Can IBD Scoring Point the Way? Br J Cancer (2020) 123 (2):207-15. doi: 10.1038/s41416-020-0882-y

43. Schroeder KW, Tremaine WJ, Ilstrup DM. Coated Oral 5-Aminosalicylic Acid Therapy for Mildly to Moderately Active Ulcerative Colitis. A Randomized Study. N Engl J Med (1987) 317(26):1625-9. doi: 10.1056/NEJM198712243172603

44. Travis SP, Schnell D, Krzeski P, Abreu MT, Altman DG, Colombel J-F, et al. Developing an Instrument to Assess the Endoscopic Severity of Ulcerative Colitis: The Ulcerative Colitis Endoscopic Index of Severity (UCEIS). Gut (2012) 61(4):535-42. doi: 10.1136/gutjnl-2011-300486

45. Bergqvist V, Hertervig E, Gedeon P, Kopljar M, Griph H, Kinhult S, et al. Vedolizumab Treatment for Immune Checkpoint Inhibitor-Induced Enterocolitis. Cancer Immunol Immunother (2017) 66(5):581-92. doi: 10.1007/s00262-017-1962-6

46. Brahmer JR, Lacchetti C, Schneider BJ, Atkins MB, Brassil KJ, Caterino J, et al. Management of Immune-Related Adverse Events in Patients Treated With Immune Checkpoint Inhibitor Therapy: American Society of Clinical Oncology Clinical Practice Guideline. J Clin Oncol (2018) 36(17):1714-68. doi: 10.1200/JCO.2017.77.6385
47. Suzuki A, Brunt EM, Kleiner DE, Miquel R, Smyrk TC, Suzuki RJ, et al. The Use of Liver Biopsy Evaluation in Discrimination of Idiopathic Autoimmune Hepatitis Versus Drug-Induced Liver Injury. Hepatology (2011) 54(3):931-9. doi: 10.1002/hep. 24481

48. Sanjeevaiah A, Kerr T, Beg MS. Approach and Management of Checkpoint Inhibitor-Related Immune Hepatitis. J Gastrointest Oncol (2018) 9(1):220-4. doi: 10.21037 /jgo.2017.08.14

49. Tarhini A. Immune-Mediated Adverse Events Associated With Ipilimumab Ctla-4 Blockade Therapy: The Underlying Mechanisms and Clinical Management. Scientifica (Cairo) (2013) 2013:857519. doi: 10.1155/2013/ 857519

50. Haanen J, Carbonne F, Robert C, Kerr KM, Peters S, Larkin PJ, et al. Management of Toxicities From Immunotherapy: ESMO Clinical Practice Guidelines for Diagnosis, Treatment and Follow-Up. Ann Oncol (2017) 28 (suppl_4):iv119-42. doi: 10.1093/annonc/mdx225

51. Ito J, Nakamura A, Nagano T, Ueharac K, Imaic Y, Tomiia K, et al. Aprepitant for Refractory Nivolumab-Induced Pruritus. Lung Cancer (2017) 109:58-61. doi: 10.1016/j.lungcan.2017.04.020

52. Naidoo J, Cappelli LC, Forde PM, Marrone KA, Lipson EJ, Hammers HJ, et al. Inflammatory Arthritis: A Newly Recognized Adverse Event of Immune Checkpoint Blockade. Oncologist (2017) 22(6):627-30. doi: 10.1634/ theoncologist.2016-0390

53. Robert C, Schachter J, Long GV, Arance A, Grob JJ, Mortier L, et al. Pembrolizumab Versus Ipilimumab in Advanced Melanoma. $N$ Engl J Med (2015) 372(26):2521-32. doi: 10.1056/NEJMoa1503093

54. Hodi FS, Lee S, McDermott DF, Rao UN, Butterfield LH, Tarhini AA, et al. Ipilimumab Plus Sargramostim $v s$ Ipilimumab Alone for Treatment of Metastatic Melanoma: A Randomized Clinical Trial. JAMA (2014) 312 (17):1744-53. doi: 10.1001/jama.2014.13943

55. Sise ME, Seethapathy H, Reynolds KL. Diagnosis and Management of Immune Checkpoint Inhibitor-Associated Renal Toxicity: Illustrative Case and Review. Oncologist (2019) 24(6):735-42. doi: 10.1634/theoncologist.20180764

56. Johnson DB, Balko JM, Pharm D, Compton ML, Chalkias S, Gorham J, et al. Fulminant Myocarditis With Combination Immune Checkpoint Blockade. N Engl J Med (2016) 375(18):1749-55. doi: 10.1056/NEJMoa1609214

57. Kennedy LC, Bhatia S, Thompson JA, Grivas P. Preexisting Autoimmune Disease: Implications for Immune Checkpoint Inhibitor Therapy in Solid Tumors. J Natl Compr Canc Netw (2019) 17(6):750-7. doi: 10.6004/ jnccn.2019.7310

58. Gutierrez-Arcelus M, Rich SS, Raychaudhuri S. Autoimmune Diseases Connecting Risk Alleles With Molecular Traits of the Immune System. Nat Rev Genet (2016) 17(3):160-74. doi: 10.1038/nrg.2015.33

59. Torkamani A, Wineinger NE, Topol EJ. The Personal and Clinical Utility of Polygenic Risk Scores. Nat Rev Genet (2018) 19(9):581-90. doi: 10.1038/ s41576-018-0018-x

60. Hasan Ali O, Berner F, Bomze D, Diem M, Cozzioe S, Jörger A, et al. Human Leukocyte Antigen Variation Is Associated With Adverse Events of Checkpoint Inhibitors. Eur J Cancer (2019) 107:8-14. doi: 10.1016/ j.ejca.2018.11.009

61. Chowell D, Krishna C, Pierini F, Makarov V, Rizvi NA, Kuo F, et al. Evolutionary Divergence of HLA Class I Genotype Impacts Efficacy of Cancer Immunotherapy. Nat Med (2019) 25(11):1715-20. doi: 10.1038/ s41591-019-0639-4

62. Chowell D, Morris LGT, Grigg CM, Weber JK, Samstein RM, Makarov V, et al. Patient HLA Class I Genotype Influences Cancer Response to Checkpoint Blockade Immunotherapy. Science (2018) 359(6375):582-7. doi: 10.1126/science.aao4572

63. Cappelli LC, Dorak MT, Bettinotti MP, Bingham COIII, Shah AA. Association of HLA-DRB1 Shared Epitope Alleles and Immune Checkpoint Inhibitor-Induced Inflammatory Arthritis. Rheumatol (Oxford) (2019) 58 (3):476-80. doi: 10.1093/rheumatology/key358

64. Inaba $\mathrm{H}$, Ariyasu $\mathrm{H}$, Iwakura $\mathrm{H}$, Ueda $\mathrm{Y}$, Kurimoto $\mathrm{C}$, Uraki $\mathrm{S}$, et al. Comparative Analysis of Human Leucocyte Antigen Between Idiopathic and Anti-PD-1 Antibody Induced Isolated Adrenocorticotropic Hormone Deficiency: A Pilot Study. Clin Endocrinol (Oxf) (2019) 91(6):786-92. doi: $10.1111 /$ cen.14082 
65. Stamatouli AM, Quandt Z, Perdigoto AL, Clark PL, Kluger H, Weiss SA, et al. Collateral Damage: Insulin-Dependent Diabetes Induced With Checkpoint Inhibitors. Diabetes (2018) 67(8):1471-80. doi: 10.2337/dbi18-0002

66. Oh DY, Cham J, Zhang L, Fong G, Kwek SS, Klinger M, et al. Immune Toxicities Elicted by CTLA-4 Blockade in Cancer Patients Are Associated With Early Diversification of the T-Cell Repertoire. Cancer Res (2017) 77 (6):1322-30. doi: 10.1158/0008-5472.CAN-16-2324

67. Kato T, Tomiyama E, Koh Y, Matsushita M, Hayashi Y, Nakano K, et al. A Potential Mechanism of Anticancer Immune Response Coincident With Immune-Related Adverse Events in Patients With Renal Cell Carcinoma. Anticancer Res (2020) 40(9):4875-83. doi: 10.21873/anticanres.14490

68. Robert L, Tsoi J, Wang X, Emerson R, Homet B, Chodon T, et al. CTLA4 Blockade Broadens the Peripheral T-Cell Receptor Repertoire. Clin Cancer Res (2014) 20(9):2424-32. doi: 10.1158/1078-0432.CCR-13-2648

69. Subudhi SK, Aparicio A, Gao J, Zurita AJ, Araujo JC, Logothetis CJ, et al. Clonal Expansion of CD8 T Cells in the Systemic Circulation Precedes Development of Ipilimumab-Induced Toxicities. Proc Natl Acad Sci USA (2016) 113(42):11919-24. doi: 10.1073/pnas.1611421113

70. Rube CE, Palm J, Erren M, Fleckenstein J, König J, Remberger K, et al. Cytokine Plasma Levels: Reliable Predictors for Radiation Pneumonitis? PloS One (2008) 3(8):e2898. doi: 10.1371/journal.pone.0002898

71. Stenmark MH, Cai X-W, Shedden K, Hayman JA, Yuan S, Ritter T, Ten RK, et al. Combining Physical and Biologic Parameters to Predict RadiationInduced Lung Toxicity in Patients With Non-Small-Cell Lung Cancer Treated With Definitive Radiation Therapy. Int J Radiat Oncol Biol Phys (2012) 84(2): e217-22. doi: 10.1016/j.jirobp.2012.03.067

72. Takahashi H, Imai Y, Fujishima T, Shiratori M, Murakami S, Chiba H, et al. Diagnostic Significance of Surfactant Proteins A and D in Sera From Patients With Radiation Pneumonitis. Eur Respir J (2001) 17(3):481-7. doi: 10.1183/ 09031936.01.17304810

73. Puzanov I, Diab A, Abdallah K, Bingham COIII, Brogdon C, Dadu R, et al. Managing Toxicities Associated With Immune Checkpoint Inhibitors: Consensus Recommendations From the Society for Immunotherapy of Cancer (SITC) Toxicity Management Working Group. J Immunother Cancer (2017) 5(1):95. doi: 10.1186/s40425-017-0300-z

74. Santini FC, Rizvi H, Plodkowski AJ, Ni A, Lacouture ME, Gambarin-Gelwan $\mathrm{M}$, et al. Safety and Efficacy of Re-Treating With Immunotherapy After Immune-Related Adverse Events in Patients With NSCLC. Cancer Immunol Res (2018) 6(9):1093-9. doi: 10.1158/2326-6066.CIR-17-0755

75. Hua C, Boussemart L, Mateus C, Routier E, Boutros C, Cazenave H, et al. Association of Vitiligo With Tumor Response in Patients With Metastatic Melanoma Treated With Pembrolizumab. JAMA Dermatol (2016) 152(1):4551. doi: $10.1001 /$ jamadermatol.2015.2707

76. Horvat TZ, Adel NG, Dang T-O, Momtaz P, Postow MA, Callahan MK, et al. Immune-Related Adverse Events, Need for Systemic Immunosuppression, and Effects on Survival and Time to Treatment Failure in Patients With Melanoma Treated With Ipilimumab at Memorial Sloan Kettering Cancer Center. J Clin Oncol (2015) 33(28):3193-8. doi: 10.1200/JCO.2015.60.8448

77. Leonardi GC, Gainor JF, Altan M, Kravets S, Dahlberg SE, Gedmintas L, et al. Safety of Programmed Death-1 Pathway Inhibitors Among Patients With NonSmall-Cell Lung Cancer and Preexisting Autoimmune Disorders. J Clin Oncol (2018) 36(19):1905-12. doi: 10.1200/JCO.2017.77.0305
78. Schadendorf D, Hodi F, Robert C, Weber JS, Margolin K, Hamid O, et al. Pooled Analysis of Long-Term Survival Data From Phase II and Phase III Trials of Ipilimumab in Unresectable or Metastatic Melanoma. J Clin Oncol (2015) 33 (17):1889-94. doi: 10.1200/JCO.2014.56.2736

79. Khan SA, Pruitt SL, Xuan L, Gerber DE. Prevalence of Autoimmune Disease Among Patients With Lung Cancer: Implications for Immunotherapy Treatment Options. JAMA Oncol (2016) 2(11):1507-8. doi: 10.1001/ jamaoncol.2016.2238

80. Coureau M, Meert A-P, Berghmans T, Grigoriu B. Efficacy and Toxicity of Immune -Checkpoint Inhibitors in Patients With Preexisting Autoimmune Disorders. Front Med (Lausanne) (2020) 7:137. doi: 10.3389/fmed.2020.00137

81. Lee B, Wong A, Kee D, Neeson N, Shackleton M, McArthur BG, et al. The Use of Ipilimumab in Patients With Rheumatoid Arthritis and Metastatic Melanoma. Ann Oncol (2016) 27(6):1174-7. doi: 10.1093/annonc/mdw056

82. Johnson DB, Sullivan RJ, Ott PA, Carlino MS, Khushalani NI, Ye F, et al. Ipilimumab Therapy in Patients With Advanced Melanoma and Preexisting Autoimmune Disorders. JAMA Oncol (2016) 2(2):234-40. doi: 10.1001/ jamaoncol.2015.4368

83. Menzies AM, Johnson DB, Atkinson VG, Wong ANM, Park JJ, McQuade JL, et al. Anti-PD-1 Therapy in Patients With Advanced Melanoma and Preexisting Autoimmune Disorders or Major Toxicity With Ipilimumab. Ann Oncol (2017) 28(2):368-76. doi: 10.1093/annonc/mdw443

84. Danlos FX, Voisin A-L, Dyevrec V, Michota J-M, Routierd E, Tailladee L, et al. Safety and Efficacy of Anti-Programmed Death 1 Antibodies in Patients With Cancer and Pre-Existing Autoimmune or Inflammatory Disease. Eur J Cancer (2018) 91:21-9. doi: 10.1016/j.ejca.2017.12.008

85. Gutzmer R, Koopa A, Meier F, Hassel JC, Terheyden P, Zimmere L, et al. Programmed Cell Death Protein-1 (PD-1) Inhibitor Therapy in Patients With Advanced Melanoma and Preexisting Autoimmunity or IpilimumabTriggered Autoimmunity. Eur J Cancer (2017) 75:24-32. doi: 10.1016/ j.ejca.2016.12.038

86. Arbour KC, Mezquita L, Long N, Rizvi H, Auclin E, Ni A, et al. Impact of Baseline Steroids on Efficacy of Programmed Cell Death-1 and Programmed Death-Ligand 1 Blockade in Patients With Non-Small-Cell Lung Cancer. J Clin Oncol (2018) 36(28):2872-8. doi: 10.1200/JCO.2018.79.0006

Conflict of Interest: The authors declare that the research was conducted in the absence of any commercial or financial relationships that could be construed as a potential conflict of interest.

Publisher's Note: All claims expressed in this article are solely those of the authors and do not necessarily represent those of their affiliated organizations, or those of the publisher, the editors and the reviewers. Any product that may be evaluated in this article, or claim that may be made by its manufacturer, is not guaranteed or endorsed by the publisher.

Copyright $\odot 2021$ Burke and Rashdan. This is an open-access article distributed under the terms of the Creative Commons Attribution License (CC BY). The use, distribution or reproduction in other forums is permitted, provided the original author $(s)$ and the copyright owner(s) are credited and that the original publication in this journal is cited, in accordance with accepted academic practice. No use, distribution or reproduction is permitted which does not comply with these terms. 